\title{
The rising applications of automation in emerging world
}

\author{
Sankar. P \\ ${ }^{1}$ Banglore University, Karnataka, India \\ Email:sankar@list.ru
}

\begin{abstract}
The emergence of automated technologies have unleashed the different roles in modern society which can be cited in every sectors in current scenario. These applications are rapidly increasing and are influencing the betterment of the society. Keeping this as consideration, the present study was designed and executed to potray the different roles.
\end{abstract}

Keywords: automation, applications, information technologies

\section{INTRODUCTION}

Security In technology field AI (Artificial intelligence) provides manufacturing applications of the automation and robotics. There are some examples for the fixed automation involving machining transfer line found in automotive industry, certain chemical process and automatic assembly machine (Meyerson, 2015). Programmable automation is the type of the developing products in the batches. Production in industry is backbone of the many world's economies, also cover the both manufacturing in factories, plants production process and research production. Due to the always growing client demands, high competiveness and the economic evaluation environment, like industries face many technological issues (Gopalakrishnan \& Biswal, 2016).

Goals for the future and present manufacturing, which are defined by the society

Technology areas where innovation is sought for, by the manufacturing industry

Factory automation requirements, to be fulfilled by the automation industry

Technology as enablers, to be investigated forward by automation research community 
Products manufacturing of the future keeps in introducing the ever changing requirements of the society when opening new markets. Personalization is a typical differentiator within higher value manufacturing this provide competitive products and new services delivery and localized functionalities. In this include new examples custom made parts and spare parts generated on demand either to the sub division of sector or product or the customized to an individual. So, order of job production is important in context of the growing (Siau, 2017). Social durability of the manufacturing introduces issue of integrating human skills with the technology. The reality of this objective forces many postulates on role of technology in future manufacturing as the follows-

To help human plant operators in handling and maintaining the production plants with the growing problem.

To help human workers while handling heavy work piece, and to advantages from experience of retire work force.

The technology areas (Ax) of mostly included in bringing out these key aims for manufacturing can be condensed in following 6 items, where like a categorize is in according to the again called roadmap.

1.Modern manufacturing process and technology, involving the integration of the design and manufacturing raw material minimization and energy consumption.

2.Mechatronics to the innovative manufacturing systems.

3.Information and communication technology.

4.Strategies of manufacturing, involving temporary utilization of production resources and cross company collaboration.

5.Workers knowledge and skills.

6.Simulation and modeling for the, product co-design, process of production and resources production.

The manufacturing changes and imposed related to the production by these technologies areas are call for new needs on the factory automation systems. Like requirements are also discussed in describes in following subsection (Carbonero, et al., 2020).

\section{Currently there are many applications for automation to the world}

There are some listed area where IoT is more awaited and companies are provide the surprise with the smart devices.

\section{Smart home}

Wouldn't, if people could switch on the air conditioning before reaching the home or to switch off the lights even after they left home. Or doors are unlock when temperory friends are access even when they are not at home. So the IoT taking the shape the companies are developing products to make people life easier and more convenient. Smart home has been become revolutionary ladder of the success in residential space and it is predict the smart homes will become as ordinary as smart phones.

\section{Wearables}

These are in explosive in demands in the markets all over the world. Some of the companies such as Google, Samsung have been invested hugely in developing these devices. These devices are installed with the software and sensors that gather data and information about users. This data is after preprocessed to retrieve important insights about the user.

\section{Connected cars}

Automotive technology has been focused on the optimizing the vehicles internally functions. however now, this is growing towards improving car in experience. Connected car is the vehicle that is capable to optimize their operations, maintenance of the car as well as comfort of the passengers utilizing onboard sensors and the internet connectivity.

\section{Industrial internet}

This is the new buzz in industrial sector, also entitled as industrial IoT. This is empowering the industrial engineering with the sensors, big data and software analytics to develop brilliant machine. IIoT 
keeps great possible quality sustainability and control. Applications to tracking real time information, tracking goods and about the inventory among retailers and suppliers automated the delivery will be improve the supply chain order.

\section{Smart cities}

This is the another IoT applications creating the curiosity among the world's population. Automated transportation, smart surveillance, water distribution, smart energy management system, envriomental monitoring and urban security these all are the examples of the IoT applications for the smart cities. Internet of things sort out the major issues which is faced through the people living in the cities such as pollution, energy shortage and traffic congestion etc. products such as cellular communication authorized smart belly trash will be send awars to the municipal services while a bin requires to be unload (Cioffi, et al., 2020).

\section{Utilization of IOT in agriculture}

As well as population is increased in the world, so the food supply is highly raised. So the governments are assisting farmers to utilization modern research and techniques to improve the production of food. Smart framing is fastest growing field in the IoT. Farmers are utilizing significant insights from data relent better return to investment. Soil moisture sensing and nutrients, handling water utilization for growing plant and determining personalize fertilizer are some easy utilization of the IoT.

\section{Smart retail}

The possible IoT in retail sector is huge. IoT gives a chance to retailers to connect with clients to improve the in store experience. Smart phone will be the method of retailers to keep connected with their clients even out of the stores. Interacting by the smart phones and utilizing Beacon technology can assist retailers serve their clients better. These can track clients path by a store and increase store layout and place the premium products in the huge traffic areas.

\section{Energy engagement}

Future power grids are will not be smart sufficient but also greatly reliable. Smart grid idea is too famous all over the world. The basic concept behind smart grid is to gather data in an automated way and examine behavior or consumers of the electricity for increasing the efficiency as well as electricity utilization economics.

\section{IOT in healthcare}

Connected with the healthcare still keeps sleeping giant of the IoT applications. The idea of the connected with the healthcare system and smart medical device bear huge possible not for the companies, however also for well being of the persons in normally

\section{IOT in poultry $\&$ farming}

Livestock analyzing is all about animal husbandry and also the cost saving. Utilizing IoT applications to collect the data about well being and health of cattle, sick animal information is provide by the ranchers and help to prevent huge number of the sick cattle. Through the help of the gathering data and ranchers can improve poultry production.

\section{Artificial intelligence}

AI (artificial intelligence) is specifically effecting the method customers interact with the businesses by the intelligent bots and websites and these all tool is becoming enhancing commoditized and unified into the regular work. And provide the report. The greatest effects over all industries like from retail to healthcare, finance to hospitality are felt while AI enhances security of the data accuracy and decision making speed and employee training and output. With the more able staff, good qualified sales leads, much efficient problem solver and system which feed real data back in for the further product and process enhancements, companies employing AI technology can utilize resources with the far huge efficiency. 


\section{G applications}

The development of the $5 \mathrm{G}$ networks is improving the ability to move, analyze, manipulate data over the wireless platforms, according to the CompTIA. As 5G roll out more completely in the coming years, this will run the development of the much complex applications to sort out issues and improve growth over the industry. Deployement and development of the $5 \mathrm{G}$ is going to authorize impact of the business at the level of few technologies ever have, giving wireless at speed and latency required for the complex solutions such as driverless vehicle, one time completely deployed geographically, 5G will assist the emerging markets realize the similar speed of the business, as their grown counterparts. Solution givers which develop 5G based solutions for the significant industry applications will have been profitable, ultimately mover benefits. 5G uses cases are-

\# Fixed wireless access

\# Video

* Immersive experience

\# Smart venues

* Connected vehicles

\section{Server less computing}

Server less computing permits companies to develop a NoOps IT environment which is automated and theoritccal from underlying the framework, minimize the operational costs and permitting businesses to invest in making innovative abilities which add more value and provide the report (Dotoli, et al., 2019).This was new to the applications list this year, along with robotics, this replaced quantum computing, and the automation. Server less computing use cases are-

* Event streaming

* Auto- scaling websites and APIs

* Video and image manipulation

* SaaS and processing events

* Hybrid cloud applications

* Multi language applications

* Continuous deployment and continuous integration

\section{Block chain}

Many of the companies are exploring and executing the block chain to sort out the improved require to protect and manage transactions over the internet. Block chain is came down squeezing from its highest hype cycle, and so likely for best. There are given below some of the applications and use cases of the block chain are-

* Applications are-

\# Secure the sharing of the medical data

* Tracking of the music royalties

* Payments of the cross border

* Real time IoT operating system

* Personal identity security

* Anti money laundering tracking system

* Logistics monitoring and supply chain

* Voting mechanism

\# Advertising insights

* Real content creation

* Cryptocurrency exchange

* Real estate processing platform 
\# Use cases are-

* Smart contracts use cases

* Money transfer use cases

* IoT (internet of things) use cases

* Personal identity security cases

* Logistics use cases

* Government use cases

* Media use cases

\section{Robotics}

Robotics is an automating regular processing through utilizing machines to develop businesses faster, more efficient, less expensive. There are robotics applications are given below-

* Collaborative robotics

* Robotic painting

* Robotic welding

* Robotic assembly

* Material removal

* Machine tending and part transfer.

\section{CONCLUSION}

The emerging roles of automations are increasing everyday and in every sector. This can be attributed to the scientific research being conducted across the globe to tap the automated processes and applications.

\section{REFERENCES}

[1] Siau, K. (2017). Impact of artificial intelligence, robotics, and automation on higher education.

[2] SURPRISES, A., Sarter, N.B., Woods, D.D., \& Billings, C.E. (2001). Automation Surprises.

[3] Parasuraman, R., Molloy, R., \& Singh, I.L. (1993). Performance Consequences of Automation-Induced 'Complacency'.

[4] Dzindolet, M.T., Peterson, S.A., Pomranky, R.A., Pierce, L.G., \& Beck, H.P. (2003). The role of trust in automation reliance. Int. J. Hum. Comput. Stud., 58, 697-718.

[5] Parasuraman, R., \& Mouloua, M. (1996). Automation and human performance : theory and applications.

[6] SM.Mohammed. (2017) .DevOps Automation and Agile Methodology. International Journal of Creative Research Thoughts (IJCRT), ISSN:2320-2882, Volume.5, Issue 3, pp.946-949,

[7] SM.Mohammed. (2016). Continuous Integration and Automation. International Journal of Creative Research Thoughts (IJCRT), ISSN:2320-2882, Volume.4, Issue 3, pp.938-945, July 2016.

[8] SM.Mohammed. (2018).Streamlining DevOps Automation For Cloud Applications. International Journal of Creative Research Thoughts (IJCRT), ISSN:2320-2882, Volume.6, Issue 4, pp.955-959, October-2018

[9] Meyerson, B. (2015, March). Top 10 emerging technologies of 2015. In World Economic Forum (Vol. 4).

[10] Gopalakrishnan, A., \& Biswal, A. C. (2016, March). Applications of emerging communication trends in automation. In 2016 IEEE 6th International Conference on Power Systems (ICPS) (pp. 1-6). IEEE. 